\title{
miR-552-5p facilitates osteosarcoma cell proliferation and metastasis by targeting WIF1
}

\author{
WEI CAI ${ }^{1}$, YONG XU ${ }^{1}$, JIAN YIN $^{2}$, WENSHAN ZUO ${ }^{1}$ and ZHEN SU ${ }^{3}$ \\ ${ }^{1}$ Orthopedics Department, The Affiliated Huaian No. 1 People's Hospital of Nanjing Medical University, Huai'an, \\ Jiangsu 223300; ${ }^{2}$ Orthopedics Department, The Affiliated Jiangning Hospital, Nanjing Medical University, Nanjing, \\ Jiangsu 211100; ${ }^{3}$ Anesthesiology Department, The Affiliated Huaian No. 1 People's Hospital of Nanjing Medical University, \\ Huai'an, Jiangsu 223300, P.R. China
}

Received June 30, 2018; Accepted December 13, 2018

DOI: $10.3892 /$ etm.2019.7361

\begin{abstract}
R-552 promotes tumor growth and metastasis in colorectal cancer. However, the function of miR-552 in osteosarcoma remains unclear. The current study investigated the role and mechanism of miR-552-5p in the proliferation, migration and invasion of osteosarcoma cells. miR-552-5p was significantly upregulated in osteosarcoma tissues and cell lines compared with adjacent normal tissues and normal osteoblast cells. Knockdown of miR-552-5p significantly reduced the proliferation of MG63 and U2OS cells, and inhibited cell migration and invasion. Wnt inhibitory factor 1 (WIF1) was the direct target gene of miR-552-5p in osteosarcoma cells. Overexpression of miR-552-5p markedly decreased the expression of WIF1 in MG63 and U2OS cells. A negative association was identified between the expression levels of miR-552-5p and WIF1 in osteosarcoma tissues. Furthermore, the expression of WIF1 was downregulated in osteosarcoma tissues and cell lines. Finally, knockdown of WIF1 in MG63 and U2OS cells treated with miR-552-5p inhibitors rescued their ability to proliferate, migrate and invade. Overall, the results indicated that miR-552-5p promoted osteosarcoma development and progression by inhibiting WIF1. Therefore, miR-552-5p may serve as a new therapeutic target for treatment of patients with osteosarcoma.
\end{abstract}

\section{Introduction}

Osteosarcoma (OS) is one of the most aggressive and common types of bone cancer and is the major cause of tumor-associated

Correspondence to: Professor Zhen Su, Anesthesiology Department, The Affiliated Huaian No. 1 People's Hospital of Nanjing Medical University, 1 Huanghe West Road, Huai'an, Jiangsu 223300, P.R. China

E-mail: suzhen_nju@163.com

Key words: microRNA-552-5p, Wnt inhibitory factor 1, proliferation, migration, osteosarcoma mortality among the youth worldwide $(1,2)$. At present, radical surgery and neo-adjuvant chemotherapy are the main methods for the treatment of OS, and these approaches markedly increase the survival of patients with OS (2). However, the clinical outcomes of OS remain poor due to the high rates of tumor metastasis and recurrence, making OS a serious threat to human health and life (3). Therefore, the underlying mechanism of OS must be elucidated to develop new effective therapeutic targets.

microRNAs (miRNAs/miRs) are a class of noncoding transcripts, 18-25 nucleotides in length, that participate in diverse human diseases, including cancer (4-6). miRNAs target the 3'-untranslated region (UTR) of specific mRNAs and regulate gene expression (7). Through this mechanism, miRNAs regulate numerous biological processes, including cell proliferation, apoptosis, differentiation and metastasis (8-10). Scholars reported that miRNAs function in different types of human cancer. For example, Bai et al (11) revealed that miRNA-196b suppressed cell metastasis and proliferation by inhibiting Runx2 in lung cancer. Wang et al (12) reported that miR-101 suppressed proliferation and migration of breast cancer cells by targeting SOX2. Furthermore, it has been demonstrated that miR-329 inhibited OS development (13), and miR-506 overexpression inhibited cell proliferation and enhanced apoptosis in OS (14). Nonetheless, the function of numerous miRNAs in OS remains unclear. Understanding the functions and regulatory networks of miRNAs may benefit OS intervention.

miR-552-5p acts as an oncogene in certain types of cancer. For instance, miRNA-552 targets dachshund homolog 1 (DACH1) to enhance cell proliferation and migration in colorectal cancer (15). Wang et al (16) revealed that miR-552 promoted colorectal cancer metastasis. Nevertheless, the role of miR-552-5p in OS remains to be investigated.

In the current study, miR-552-5p was overexpressed in OS tissues and cell lines compared with normal tissues and osteoblast cells. Knockdown of miR-552-5p significantly suppressed the proliferation, migration and invasion of OS cells. miR-552-5p inhibitors inhibited the cell cycle and it directly targeted the 3'-UTR of Wnt inhibitory factor 1 (WIF1) mRNA. Furthermore, miR-552-5p overexpression inhibited the WIF1 expression in OS cells. WIF1 was downregulated 
in OS tissues and cell lines. WIF1 is an inhibitor of the $\mathrm{Wnt} / \beta$-catenin pathway, which serves a role in tumor growth and metastasis (17). By inhibiting WIF1 expression, miR-552-5p promoted the proliferation, migration and invasion of OS cells.

\section{Materials and methods}

Clinical specimens. A total of 51 histologically diagnosed OS tissues (33 patients $<20$ years old and 18 patients $\geq 20$ years old; sex: 30 males and 21 females) and 19 adjacent normal tissues were collected from patients of the Huai'an First People's Hospital, Nanjing Medical University (Huai'an, China). The samples were divided into two groups according to the existence of lymph node metastasis. None of the patients received immunotherapy, radiotherapy or chemotherapy prior to the surgery. Written informed consent was obtained from each patient before the clinical specimens were used. The tissue specimens were conserved in liquid nitrogen for further investigation. The present study was approved by the Research Ethics Committee of Nanjing Medical University in accordance with the Declaration of Helsinki.

Cell culture and transfection. Human osteoblast cell line hFOB1.19 (CRL-11372 ${ }^{\mathrm{TM}}$ ) and human OS cell lines, including U2OS, MG63 and SAOS2 were obtained from the American Type Culture Collection (Manassas, VA, USA). The cells were cultured in Dulbecco's modified Eagle's medium (DMEM; HyClone; GE Healthcare Life Sciences, Logan, UT, USA) supplemented with $10 \%$ fetal bovine serum (FBS; HyClone; GE Healthcare Life Sciences) and penicillin and streptomycin (both $50 \mathrm{U} / \mathrm{ml}$; Thermo Fisher Scientific, Inc., Waltham, MA, USA) in a humidified incubator with $5 \% \mathrm{CO}_{2}$ at $37^{\circ} \mathrm{C}$.

miR-552-5p inhibitors (50 nM; 5'-CCAACAGGCAAA AGGUUAAAC-3'), miR-552-5p mimics (50 nM; 5'-GUU UAACCUUUUGCCUGUUGG-3') and negative controls (NC; 50 nM; 5'-UCACAACCUCCUAGAAAGAGUAGA-3') were obtained from GeneCopoeia, Inc. (Rockville, MD, USA). Specific small interfering (si)RNAs against WIF1 (100 nM; siWIF1, 5'-GCA AUAUAAUAUAUUGUA AAC-3') and scrambled NC (100 nM; siNC, 5'-AAUUCU CCGAACGUGUCACGU-3') were synthesized by Wuhan Genesil Biotechnology Co., Ltd (Wuhan, China). miRNAs and siRNAs were transfected into $5 \times 10^{6}$ cells using Lipofectamine ${ }^{\circledR} 2000$ (Invitrogen; Thermo Fisher Scientific, Inc.) in accordance with the manufacturer's protocol. A total of $48 \mathrm{~h}$ post transfection, the efficiency was validated using Reverse transcription-quantitative polymerase chain reaction (RT-qPCR).

Cell proliferation assay. Cell proliferation was detected using a Cell Counting Kit-8 (CCK-8; Dojindo Molecular Technologies, Inc., Kumamoto, Japan). The cells were grown in a 96-well plate at a density of $2 \times 10^{3}$ cells/well and incubated at $37^{\circ} \mathrm{C}$ under $5 \% \mathrm{CO}_{2}$ until a cell confluence rate of $70 \%$ was attained. Following transfection with the miRNA inhibitors or NC for $48 \mathrm{~h}$, the cells were cultured for 24,48 and $72 \mathrm{~h}$. Then, CCK-8 solution, $\sim 10 \mu \mathrm{l}$, was added into each well. The absorbance was measured at a wavelength of $450 \mathrm{~nm}$ using a
Sunrise $^{\mathrm{TM}}$ microplate reader (Tecan Group, Ltd., Mannedorf, Switzerland).

Colony formation assay. For the colony formation assay, OS cells were counted at $24 \mathrm{~h}$ post-transfection and seeded into 24-well plates at a density of $1 \times 10^{3}$ cells/well. The culture medium was replaced every 3 days. After 14 days, the cells were washed with $1 \mathrm{X}$ PBS, fixed with $4 \%$ formaldehyde for $30 \mathrm{~min}$ at $25^{\circ} \mathrm{C}$, stained with $0.5 \%$ crystal violet for $30 \mathrm{~min}$ at $25^{\circ} \mathrm{C}$, and counted using an inverted light microscope (IX83; Olympus Corporation, Tokyo, Japan) at a magnification of x100.

Migration and invasion assay. The lower chambers $(8 \mu \mathrm{m}$ pore; Corning, Incorporated, Corning, NY, USA) were filled with $600 \mu 1$ of DMEM supplemented with $20 \%$ FBS to induce the migration of OS cells. A total of $2 \times 10^{4}$ cells were seeded in the upper chamber filled with $200 \mu 1$ serum-free DMEM medium. At $24 \mathrm{~h}$ after seeding, OS cells that migrated through the membranes were stained with $0.5 \%$ crystal violet dye for $30 \mathrm{~min}$ at $25^{\circ} \mathrm{C}$ and cell number was counted under the light microscope at a magnification of $\times 100$. For the invasion assay, the membrane was covered with $70 \mu \mathrm{l}$ of Matrigel and the remaining steps were the same as for the migration assay.

$R T-q P C R$. RNA was isolated from $1 \times 10^{7}$ cells using TRIzol ${ }^{\circledR}$ reagent (Invitrogen; Thermo Fisher Scientific, Inc.) in accordance with the manufacturer's protocol. First-strand cDNA was synthesized using a TIANScript RT kit (Tiangen Biotech Co., Ltd., Beijing, China). The thermocycling conditions were as follows: $37^{\circ} \mathrm{C}$ for $25 \mathrm{~min}$ and $85^{\circ} \mathrm{C}$ for 5 sec. qPCR amplification was performed using TaqMan Human microRNA assay (Applied Biosystems; Thermo Fisher Scientific, Inc.) for miRNA analysis and UltraSYBR Mixture (cat. no. CW0957; Beijing ComWin Biotech, Co., Ltd., Beijing, China) for mRNA analysis on a LightCycler ${ }^{\circledR} 480$ PCR system (Roche Diagnostics, Indianapolis, IN, USA). The thermocycling conditions were as follows: Denaturation at $95^{\circ} \mathrm{C}$ for $10 \mathrm{~min}$, followed by 40 cycles of denaturation at $95^{\circ} \mathrm{C}$ for $15 \mathrm{sec}$ and elongation at $60^{\circ} \mathrm{C}$ for $1 \mathrm{~min}$. The gene expression was calculated using the $2^{-\Delta \Delta \mathrm{Cq}}$ method (18). U6 was used as a reference gene to normalize the miR-552-5p expression. The primers used for miR-552-5p and U6 were synthesized and purchased from GeneCopoeia, Inc. (Rockville, MD, USA). GAPDH was used to normalize WIF1 expression. The primer sequences were as follows: WIF1 (forward, 5'-TTGTTTCAGTGCTTT GGGACAG-3' and reverse, 5'-CCCCCAGACACCATAAAT GC-3'), miR-552-5p (forward, 5'-GTTTAACCTTTTGCCTGT TGG-3' and reverse, 5'-CGAACGCTTCACGAATTTG-3'), U6 (forward, 5'-ATTGGAACGATACAGAGAAGATT-3' and reverse, 5'-CGAACGCTTCACGAATTTG-3') and GAPDH (forward, 5'-TCATGGGTGTGA ACCATGAGAA-3' and reverse, 5'-GGCATGGACTGTGGTCATGAG-3').

Luciferase reporter assay. The potential targets of miR-552-5p were analyzed using the TargetScan7 tool (http://www. targetscan.org/vert_71/). The wild-type (WT) or mutant (MUT) 3'-UTR of WIF1 was amplified and cloned into a pmiR-RB-REPORT ${ }^{\mathrm{TM}}$ luciferase vector (Promega Corporation, Madison, WI, USA). The WT or MUT 3'-UTR of WIF1 was co-transfected with a miR-552-5p mimic or 
negative control into $5 \times 10^{3}$ cells using Lipofectamine ${ }^{\circledR} 2000$ (Invitrogen; Thermo Fisher Scientific, Inc.). A total of $48 \mathrm{~h}$ after the co-transfection, reporter activity was detected using a Dual-Luciferase ${ }^{\circledR}$ Reporter Assay kit (Promega Corporation). The firefly luciferase activity was normalized to the luciferase activity of Renilla.

Cell cycle analysis. A total of $1 \times 10^{6}$ cells were harvested, washed twice with ice-cold PBS and then fixed in $70 \%$ ethanol for $24 \mathrm{~h}$ at $4^{\circ} \mathrm{C}$. Cells were subsequently washed three times with ice cold PBS and incubated with $1 \mathrm{mg} / \mathrm{ml}$ RNase A (cat. no. R6148; Sigma-Aldrich; Merck KGaA, Darmstadt, Germany) for $30 \mathrm{~min}$ at $37^{\circ} \mathrm{C}$. Following this, cells were stained at $25^{\circ} \mathrm{C}$ for $10 \mathrm{~min}$ with $50 \mu \mathrm{g} / \mathrm{ml}$ propidium iodide (BD Biosciences, Franklin Lakes, NJ, USA) in 0.5\% Tween-20 with PBS and subjected to analysis of cell cycle distribution using a BD FACScan flow cytometer coupled with Cell Quest acquisition and analysis programs (version 2; both BD Biosciences).

Western blotting. Cells were lysed in cold radioimmunoprecipitation assay buffer (Thermo Fisher Scientific, Inc.) and the protein concentration was determined using a Bicinchoninic Acid Protein Assay kit (Pierce; Thermo Fisher Scientific, Inc.). Protein (40 $\mu \mathrm{g} /$ lane) was separated via SDS-PAGE on a $10 \%$ gel and then transferred to a polyvinylidene difluoride (PVDF) membrane (Thermo Fisher Scientific, Inc.). Following this, the membrane was blocked using 5\% non-fat milk in PBS (Thermo Fisher Scientific, Inc.) containing 0.1\% Tween-20 (Sigma-Aldrich; Merck KGaA) at room temperature for $3 \mathrm{~h}$. Subsequently, the PVDF membrane was incubated with anti-Cyclin D1 (cat. no. ab16663), anti- proliferating cell nuclear antigen (PCNA; cat. no. ab92552), anti-p21 (cat. no. ab109520) and anti-GAPDH (cat. no. ab9485; all 1:1,000; Abcam, Cambridge, MA, USA) primary antibodies at room temperature for $2 \mathrm{~h}$. Following washing with PBS for $10 \mathrm{~min}$, the PVDF membrane was incubated with horseradish peroxidase-conjugated goat anti-rabbit secondary antibodies (cat. no. ab7090; 1:5,000; Abcam) at room temperature for $1 \mathrm{~h}$. Membranes were then washed with PBS for 10 min and the protein bands were visualized using an Enhanced Chemiluminescence Western Blotting kit (Pierce; Thermo Fisher Scientific, Inc.) in accordance with the manufacturer's protocol. Protein densitometry was performed using ImageJ software (version 1.41; National Institutes of Health, Bethesda, MD, USA).

Statistical analysis. Data are presented as the mean \pm standard deviation and were analyzed using GraphPad Prism 5 software (GraphPad Software, Inc., La Jolla, CA, USA). Pearson's correlation coefficient analysis was used to determine association between miR-552-5p and WIF1 expression. Student's t-test and one-way analysis of variance followed by Tukey's post hoc test were used to analyze two or multiple groups, respectively. $\mathrm{P}<0.05$ was considered to indicate a statistically significant difference.

\section{Results}

miR-552-5p is overexpressed in osteosarcoma tissues and cell lines. To investigate the function of miR-552-5p, the

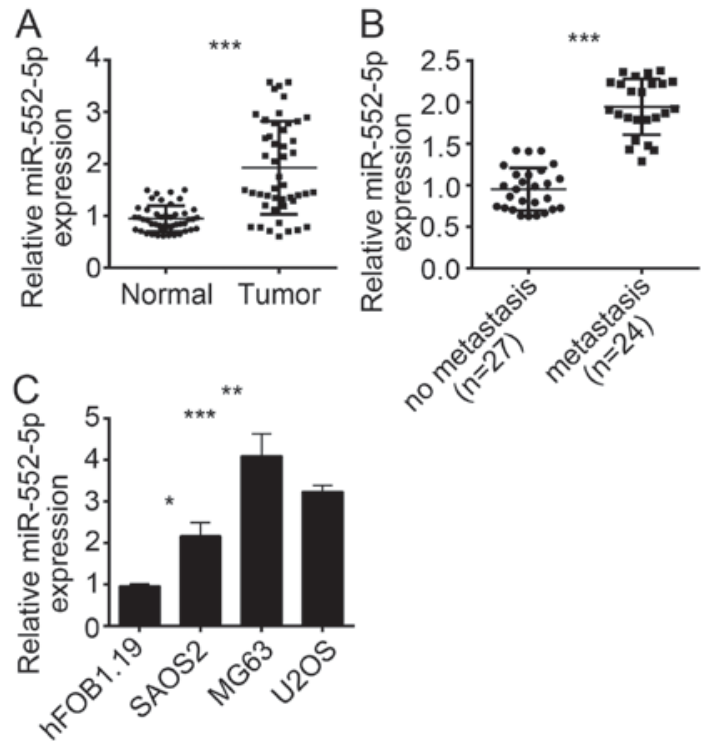

Figure 1. miR-552-5p is overexpressed in osteosarcoma tissues and cell lines. (A) Reverse transcription-quantitative polymerase chain reaction was used to determine the expression of miR-552-5p in osteosarcoma tissues $(n=51)$ and adjacent normal tissues $(n=19)$. (B) Expression of miR-552-5p was assessed in metastatic and non-metastatic osteosarcoma tissues. (C) Expression of miR-552-5p was assessed in osteosarcoma cell lines, including SAOS2, MG63 and U2OS cells, and normal osteoblast hFOB1.19 cells. * $\mathrm{P}<0.05$, ${ }^{* *} \mathrm{P}<0.01$ and ${ }^{* * * *} \mathrm{P}<0.001$. miR, microRNA.

expression of this miRNA was initially determined in OS tissues. The results of RT-qPCR revealed that the expression of miR-552-5p was upregulated in OS tissues compared with adjacent normal tissues (Fig. 1A). OS metastasis is a major cause of OS-induced mortality and poor outcomes (13). To determine the association between miR-552-5p expression and OS metastasis, the 51 OS tissues were divided into the metastasis and non-metastasis groups. The RT-qPCR result indicated that miR-552-5p exhibited an increased expression level in metastatic OS tissues compared with non-metastatic tissues (Fig. 1B). The expression pattern of miR-552-5p was further determined in OS cell lines. The results revealed that miR-552-5p was overexpressed in SAOS2, MG63 and U2OS cells compared with normal osteoblast hFOB1.19 cells (Fig. 1C). The miR-552-5p expression was the highest in MG63 and U2OS cells. Therefore, MG63 and U2OS cells were used in the subsequent experiments.

miR-552-5p knockdown suppresses proliferation, migration and invasion of MG63 and U2OS cells. The expression of miR-552-5p was knocked down by miR-552-5p inhibitors in MG63 and U2OS cells (Fig. 2A). To evaluate the effect of miR-552-5p on cell proliferation, CCK-8 and colony formation assays were performed in MG63 and U2OS cells treated with miR-552-5p inhibitor or NC. The results indicated that miR-552-5p knockdown significantly inhibited cell proliferation and colony formation (Fig. 2B and C). As aforementioned, metastatic OS tissues exhibited a high miR-552-5p expression (Fig. 1B). Therefore, the effect of miR-552-5p on cellular mobility was further assessed in vitro. Transwell assays revealed that miR-552-5p knockdown markedly suppressed the migration and invasion of MG63 and U2OS cells, compared with the corresponding NC-inhibitor groups (Fig. 2D and E). 

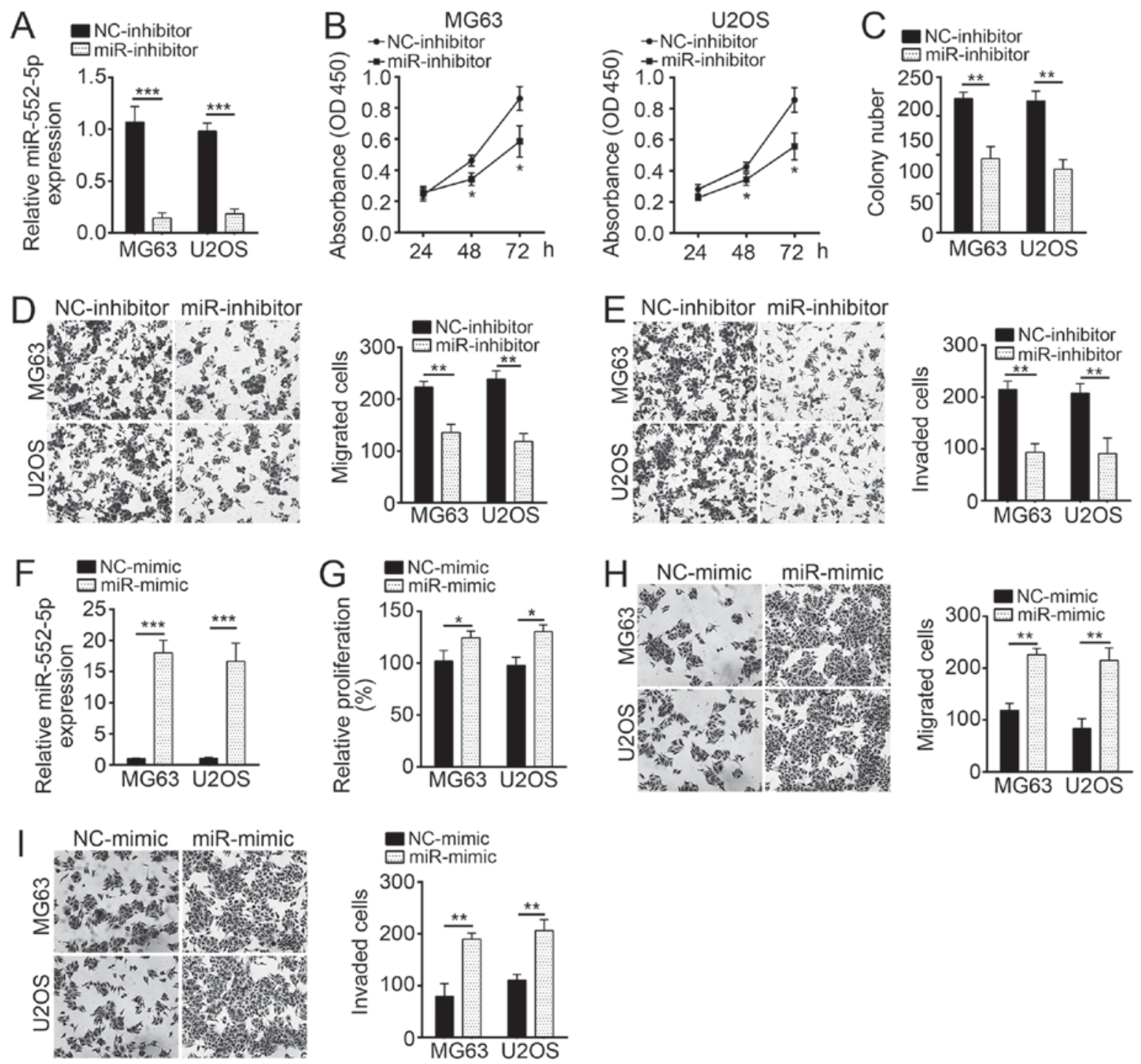

Figure 2. Knockdown of miR-552-5p suppresses proliferation, migration and invasion of MG63 and U2OS cells. (A) Reverse transcription-quantitative polymerase chain reaction analysis revealed that the expression level of miR-552-5p decreased in MG63 and U2OS cells following transfection with miR-552-5p inhibitors, compared with the NC-inhibitor group. Results of (B) CCK-8 and (C) colony formation assays indicated that miR-552-5p knockdown inhibited the proliferation of MG63 and U2OS cells. Transwell assays indicated that miR-552-5p knockdown significantly reduced (D) migration and (E) invasion of MG63 and U2OS cells (magnification, x100). (F) Expression levels of miR-552-5p were upregulated following transfection with miR-552-5p mimics in OS cells. CCK-8 and Transwell assays revealed that overexpression of miR-552-5p promoted (G) proliferation, $(\mathrm{H})$ migration and (I) invasion of OS cells. "P<0.05, ${ }^{* *} \mathrm{P}<0.01$ and ${ }^{* * *} \mathrm{P}<0.001$ vs. the respective NC group. miR, microRNA; CCK-8, Cell Counting Kit-8; NC, negative control.

Furthermore, ectopic expression of miR-552-5p promoted proliferation, migration and invasion of OS cells, compared with the NC-mimic treatment (Fig. 2F-I).

miR-552-5p knockdown inhibits the cell cycle of osteosarcoma cells. To investigate the mechanism underlying the decreased cell proliferation following transfection with miR-552-5p inhibitors, the cell cycle of MG63 and U2OS cells was analyzed. Following transfection with miR-552-5p inhibitors, a significantly increased number of MG63 and U2OS cells remained in the G0 phase, compared with the $\mathrm{NC}$ inhibitor group. Furthermore, an increased number of cells in the NC inhibitor group entered the $\mathrm{S}$ and $\mathrm{G} 2 / \mathrm{M}$ phases, compared with cells treated with miR-552-5p inhibitors (Fig. 3A and B). Protein expression levels of cell cycle-associated genes, including Cyclin D1, PCNA and p21 (4), were determined through western blot analysis. Following miR-552-5p knockdown, the expression levels of Cyclin D1 and PCNA were decreased, whereas the expression of $\mathrm{p} 21$, a cell-cycle inhibitor (19), was increased, compared with the NC inhibitor group (Fig. 3C).

WIF1 is a target of miR-552-5p in osteosarcoma cells. miRNAs regulate gene expression by targeting the 3'-UTR of specific mRNAs (20). To determine the molecular mechanism of miR-552-5p, downstream target genes of miR-552-5p were analyzed using bioinformatics methods. The results of this analysis indicated that WIF1 was a potential target of miR-552-5p, as this miRNA included a potential binding site in the 3'-UTR of WIF1 mRNA (Fig. 4A). Luciferase activity reporter assay indicated that the transfection with miR-552-5p mimics inhibited luciferase intensity in MG63 and U2OS cells transduced with the WT 3'-UTR of WIF1 mRNA (Fig. 4B). However, the mutation of the binding site of miR-552-5p in 
A

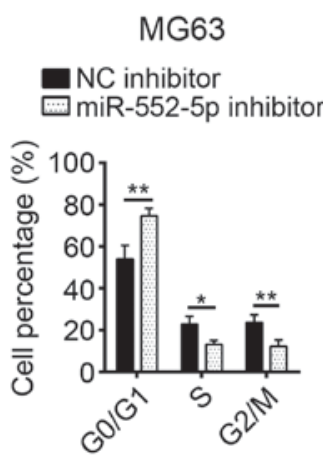

B

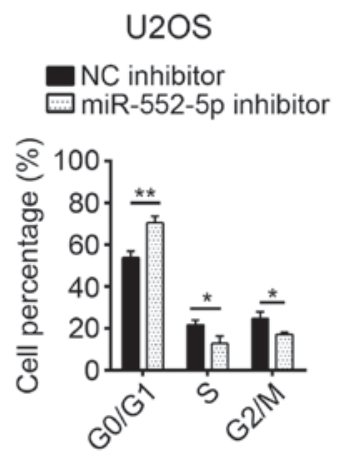

C

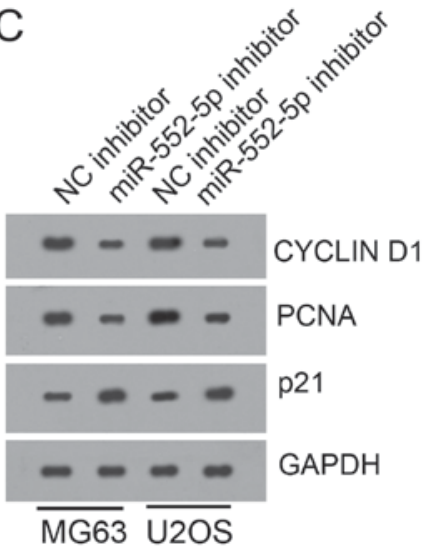

Figure 3. miR-552-5p knockdown inhibits the cell cycle of osteosarcoma cells. Cell cycle analysis of (A) MG63 and (B) U2OS cells. (C) miR-552-5p knockdown inhibited the expression of Cyclin D1 and PCNA, and promoted the expression of p21 in MG63 and U2OS cells. ${ }^{*} \mathrm{P}<0.05$ and ${ }^{* *} \mathrm{P}<0.01$. miR, microRNA; NC, negative control; PCNA, proliferating cell nuclear antigen.

\section{A}

WIF1 WT: 5'-UAUGUCAAUUGAUCAGGUUAAAA- 3 ' miR-552-5p: 3'-GGUUGUCCGUUUUCCAAUUUG-5'

WIF1 MUT: 5'-UAUGUCAAUUGAUCUCCAAUUUA-3'
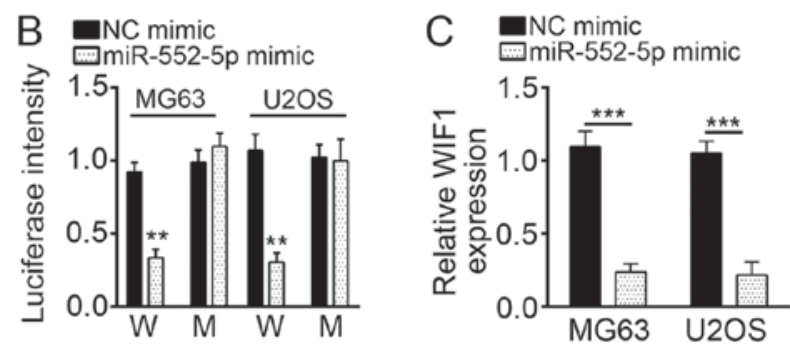
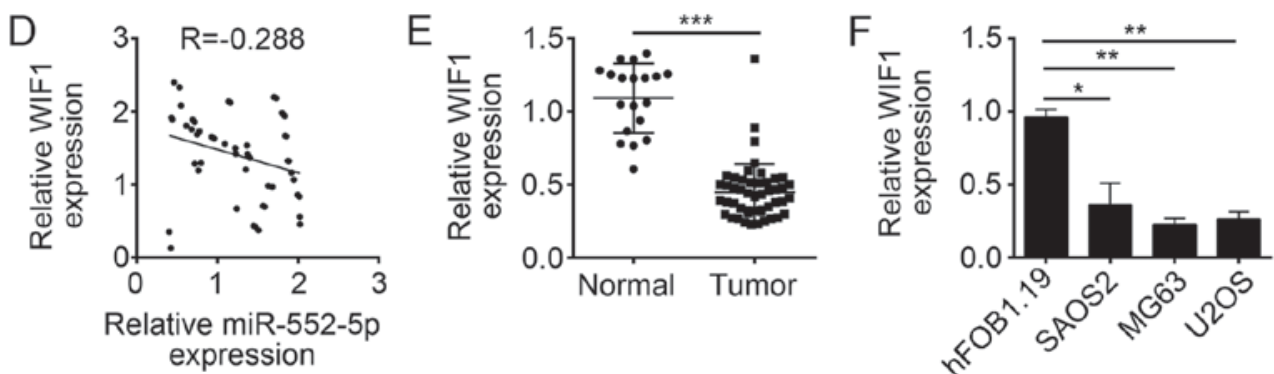

Figure 4. WIF1 is a target of miR-552-5p in osteosarcoma cells. (A) miR-552-5p may bind to the 3'-UTR of WIF1 mRNA. The underlined sequence is the mutated site. (B) miR-552-5p mimics inhibited the luciferase intensity in MG63 and U2OS cells while mutation of associating element in 3'-UTR of WIF1 mRNA abolished the effect of miR-552-5p mimic on luciferase intensity. (C) Overexpression of miR-552-5p decreased the mRNA expression level of WIF1 in MG63 and U2OS cells. (D) Expression of miR-552-5p was inversely associated with that of WIF1 in osteosarcoma tissues. (E) Reverse transcription-quantitative polymerase chain reaction analysis indicated that the expression of WIF1 was downregulated in the tumor group (n=51) compared with adjacent normal tissues ( $\mathrm{n}=19)$. (F) Expression of WIF1 was downregulated in osteosarcoma cell lines SAOS2, U2OS and MG63 cells, compared with hFOB1.19 cells. "P<0.05, ${ }^{* *} \mathrm{P}<0.01$ and ${ }^{* * *} \mathrm{P}<0.001$. miR, microRNA; NC, negative control; WIF1, Wnt inhibitory factor 1; UTR, untranslated region; WT or W, wild-type 3'-UTR; MUT or M, mutant 3'-UTR; tumor, osteosarcoma tissues.

the 3'-UTR of WIF1 mRNA abrogated the inhibitory effect of miR-552-5p mimics on the luciferase intensity (Fig. 4B). These data implied that miR-552-5p directly targeted the mRNA of WIF1 in MG63 and U2OS cells. Furthermore, overexpression of miR-552-5p significantly downregulated the mRNA expression level of WIF1 in MG63 and U2OS cells (Fig. 4C). In addition, the expression levels of miR-552-5p and WIF1 were inversely correlated in OS tissues (Fig. 4D). The WIF1 expression in OS tissues and cell lines was determined by RT-qPCR. WIF1 was significantly downregulated in OS tissues and cell lines compared with normal tissues and cells (Fig. 4E and F).

Knockdown of WIF1 in MG63 and U2OS cells treated with miR-552-5pR inhibitor rescues cell proliferation, migration, and invasion. To verify whether the regulation of OS cells by miR-552-5p was dependent on WIF1, MG63 and U2OS cells transfected with miR-552-5p inhibitors were treated with si-WIF1, which rescued the expression of WIFI to the control level (Fig. 5A). CCK-8 and Transwell assays were used to evaluate the effects of miR-552-5p and WIF1 on the proliferation, migration, and invasion of cells. Knockdown of miR-552-5p inhibited the proliferation, migration, and invasion of MG63 and U2OS cells (Fig. 5B-D). However, simultaneous knockdown of miR-552-5p and WIF1 rescued the proliferation, migration, and invasion of MG63 and U2OS cells to the control level (Fig. 5B-D). It may be concluded that miR-552-5p regulated the proliferation, migration, and invasion of OS cells by targeting WIF1. 


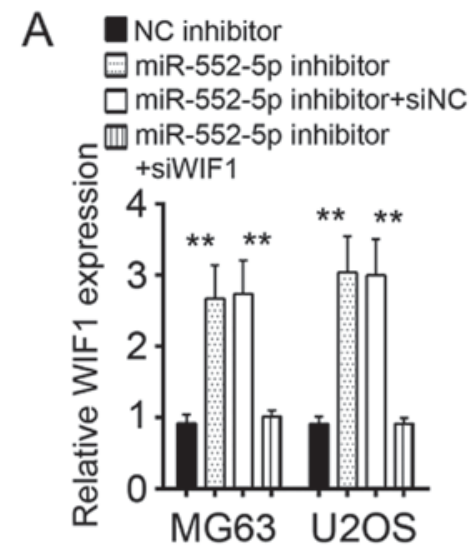

C

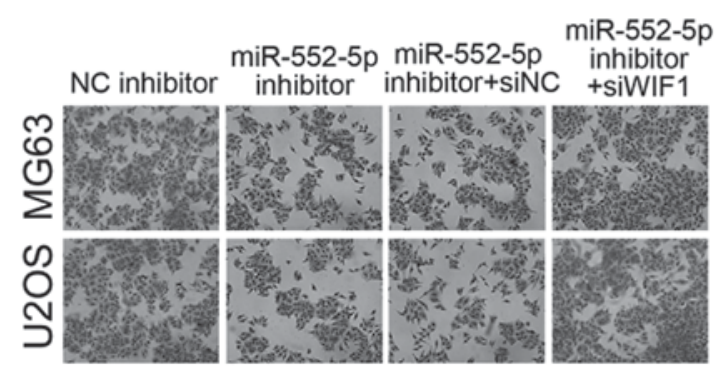

D

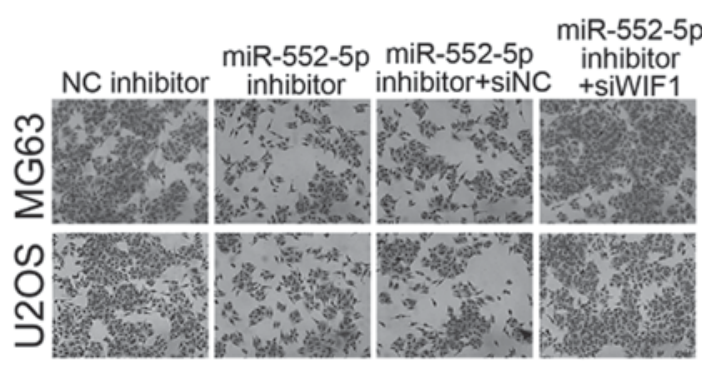

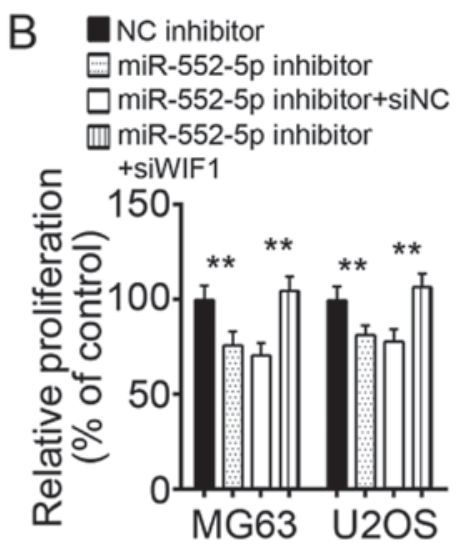
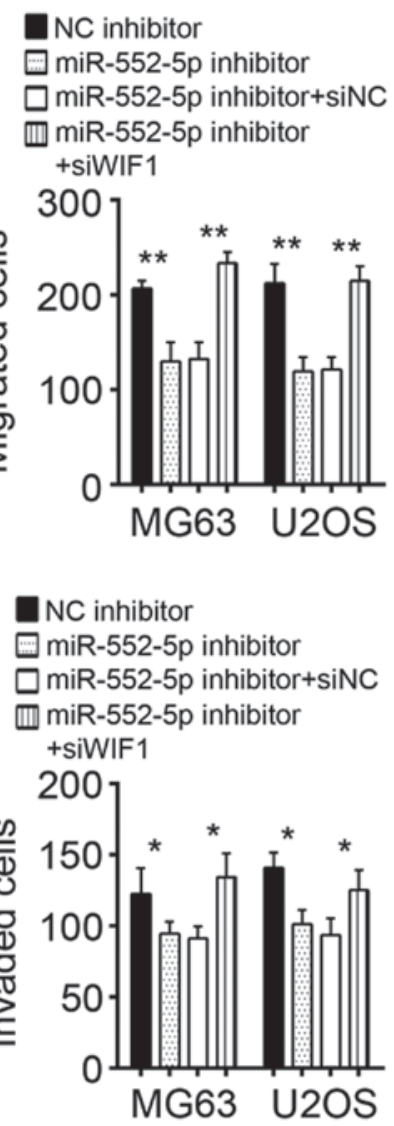

Figure 5. Knockdown of WIF1 in miR-552-5p-silenced MG63 and U2OS cells rescued cell proliferation, migration and invasion. (A) Reverse transcription-quantitative polymerase chain reaction revealed that WIF1 expression was restored to the control level in MG63 and U2OS cells with miR-552-5p knockdown. (B) Cell Counting Kit-8 results indicated that knockdown of WIF1 rescued the proliferation ability of MG63 and U2OS cells. Transwell assays demonstrated that knockdown of WIF1 rescued the $(\mathrm{C})$ migration and (D) invasion ability of MG63 and U2OS cells. ${ }^{*} \mathrm{P}<0.05$ and ${ }^{* *} \mathrm{P}<0.01$. miR, microRNA; NC, negative control; WIF1, Wnt inhibitory factor 1; siWIF1, small interfering RNA targeting WIF1.

\section{Discussion}

OS is the most malignant bone cancer worldwide and is associated with poor clinical outcomes due to tumor metastasis and recurrence (21). Therefore, research into the molecular mechanism that regulates OS occurrence and progression is required in order to screen new effective therapeutic targets. In the current study, miR-552-5p was overexpressed in OS tissues and cell lines compared with normal tissues and cells. In addition, miR-552-5p promoted the proliferation, migration and invasion of OS cells, suggesting that miR-552-5p may be a novel target for OS therapy.
A previous study has demonstrated that the dysregulation of miRNAs, which target specific genes to regulate their expression, was associated with different types of human cancer (22). Increasing number of studies indicate that miRNAs regulate the proliferation, apoptosis and metastasis of various types of cancer cells $(23,24)$. miR-124 suppresses the invasion and epithelial-mesenchymal transition of gastric cancer cells by inhibiting snail family transcriptional repressor 2 (23). Tang et al (25) reported that miR-29a inhibited cell proliferation, migration and invasion in esophageal squamous cell carcinoma. Liu et al (26) demonstrated that miR-1297 promoted breast cancer growth by activating the phosphatase 
and tensin homolog/phosphoinositide 3-kinase/protein kinase B signaling pathway. miRNA-552 promotes growth and metastasis of colorectal cancer cells (15). Cao et al (15) revealed that miR-552 targeted DACH1 and regulate the Wnt/ $\beta$-catenin pathway in colorectal cancer. Wang et al (16) demonstrated that miR-552 targeted ADAM metallopeptidase domain 28 to enhance metastasis in colorectal cancer. In the present study, CCK-8 and Transwell assays revealed that miR-552-5p promoted proliferation, migration and invasion of tumor cells in vitro.

The Wnt/ $\beta$-catenin pathway regulates numerous cellular processes, including cell proliferation, metastasis and differentiation $(27,28)$. Abnormal activation of the $\mathrm{Wnt} / \beta$-catenin pathway promotes OS development and progression (29). WIF1 serves as a regulator of Wnt/ $\beta$-catenin signaling (30). WIF1 inhibits activation of the Wnt signaling pathway by associating with Wnt proteins (31). Furthermore, WIF1 inhibits the progression of various types of cancer, including colon cancer, non-small cell lung cancer and endometrial adenocarcinoma $(20,31,32)$. A previous report indicated that WIF1 suppresses OS development and metastasis (33). In the present study, miR-552-5p directly targeted the 3'-UTR of WIF1 mRNA in OS cells. RT-qPCR results revealed an inverse correlation between the expression levels of miR-552-5p and WIF1 in OS tissues. The WIF1 expression was downregulated in OS tissues and cells. To determine whether the promotion of OS progression by miR-552-5p was dependent on WIF1, WIF1 and miR-552-5p were knocked down in MG63 and U2OS cells. The results of the CCK-8 and Transwell assays revealed that WIF1 knockdown rescued proliferation, migration, and invasion of OS cells with downregulated miR-552-5p expression. However, further investigation is required to confirm whether miR-552-5p overexpression promotes activation of the Wnt/ $\beta$-catenin signaling pathway in OS cells.

In conclusion, the present study demonstrated that miR-552-5p promoted proliferation, migration and invasion of OS cells by targeting WIF1. Therefore, the miR-552-5p/WIF1 signaling may serve as a therapeutic target for OS treatment.

\section{Acknowledgements}

Not applicable.

\section{Funding}

No funding was received.

\section{Availability of data and materials}

All data generated or analyzed during this study are included in this published article.

\section{Authors' contributions}

ZS and WC initiated and designed this study, analyzed and interpreted the results and wrote the manuscript. WC participated in all the experiments. YX,JY and WZ performed western blotting. All authors read and approved the final manuscript.

\section{Ethics approval and consent to participate}

The present study was approved by the Research Ethics Committee of Nanjing Medical University (Nanjing, China) and all enrolled patients signed a written informed consent document.

\section{Patient consent for publication}

All patients included in this study provided consent for the publication of their data.

\section{Competing interests}

The authors declare that they have no competing interests.

\section{References}

1. Yu JJ, Pi WS, Cao Y, Peng AF, Cao ZY, Liu JM, Huang SH, Liu ZL and Zhang W: Let-7a inhibits osteosarcoma cell growth and lung metastasis by targeting Aurora-B. Cancer Manag Res 10: 6305-6315, 2018.

2. Zheng WP, Huang YP, Chen HY, Wang N, Xiao W, Liang Y, Jiang $X$, Su W and Wen S: Nomogram application to predict overall and cancer-specific survival in osteosarcoma. Cancer Manag Res 10: 5439-5450, 2018.

3. Pan R, He Z, Ruan W, Li S, Chen H, Chen Z, Liu F, Tian X and Nie Y: IncRNA FBXL19-AS1 regulates osteosarcoma cell proliferation, migration and invasion by sponging miR-346. Onco Targets Ther 11: 8409-8420, 2018.

4. Liu M, Lang N, Qiu M, Xu F, Li Q, Tang Q, Chen J, Chen X, Zhang S, Liu Z, et al: miR-137 targets Cdc 42 expression, induces cell cycle G1 arrest and inhibits invasion in colorectal cancer cells. Int J Cancer 128: 1269-1279, 2011.

5. Mei Q, Li X, Guo MZ, Fu XB and Han WD: The miRNA network: micro-regulator of cell signaling in cancer. Expert Rev Anticancer Ther 14: 1515-1527, 2014.

6. Bartel DP: MicroRNAs: Genomics, biogenesis, mechanism, and function. Cell 116: 281-297, 2004.

7. Kushlinskii NE, Fridman MV and Braga EA: Molecular mechanisms and microRNAs in osteosarcoma pathogenesis. Biochemistry (Mosc) 81: 315-328, 2016.

8. Calin GA and Croce CM: MicroRNA signatures in human cancers. Nature Reviews Cancer 6: 857-866, 2006.

9. Lu J, Getz G, Miska EA, Alvarez-Saavedra E, Lamb J, Peck D, Sweet-Cordero A, Ebert BL, Mak RH, Ferrando AA, et al: Microrna expression profiles classify human cancers. Nature 435: 834-8, 2005.

10. Kim KJ and Cho SB: Exploring features and classifiers to classify microrna expression profiles of human cancer. Neural Inf Process Models Appl 6444: 234-241, 2010.

11. Bai X, Meng L, Sun H,LiZ, Zhang X and Hua S: MicroRNA-196b inhibits cell growth and metastasis of lung cancer cells by targeting Runx2. Cell Physiol Biochem 43: 757-767, 2017.

12. Wang J, Zeng H, Li H, Chen T, Wang L, Zhang K, Chen J, Wang R, Li Q and Wang S: MicroRNA-101 inhibits growth, proliferation and migration and induces apoptosis of breast cancer cells by targeting sex-determining region Y-Box 2. Cell Physiol Biochem 43: 717-732, 2017.

13. Jiang W, Liu J, Xu T and Yu X: MiR-329 suppresses osteosarcoma development by downregulating Rab10. FEBS Lett 590: 2973-2981, 2016.

14. Yao J, Qin L, Miao S, Wang X and Wu X: Overexpression of miR-506 suppresses proliferation and promotes apoptosis of osteosarcoma cells by targeting astrocyte elevated gene-1. Oncol Lett 12: 1840-1848, 2016.

15. Cao J, Yan XR, Liu T, Han XB, Yu JJ, Liu SH and Wang LB: MicroRNA-552 promotes tumor cell proliferation and migration by directly targeting DACH1 via the Wnt $/ \beta$-catenin signaling pathway in colorectal cancer. Oncol Lett 14: 3795-3802, 2017.

16. Wang J, Li H, Wang Y, Wang L, Yan X, Zhang D, Ma X, Du Y, Liu X and Yang Y: MicroRNA-552 enhances metastatic capacity of colorectal cancer cells by targeting a disintegrin and metalloprotease 28. Oncotarget 7: 70194-70210, 2016. 
17. Luo X, Ye S, Jiang Q, Gong Y, Yuan Y,Hu X, Su X and Zhu W: Wnt inhibitory factor-1-mediated autophagy inhibits Wnt/ $\beta$-catenin signaling by downregulating dishevelled-2 expression in non-small cell lung cancer cells. Int J Oncol 53: 904-914, 2018.

18. Livak KJ and Schmittgen TD: Analysis of relative gene expression data using real-time quantitative PCR and the 2(-Delta Delta C(T)) method. Methods 25: 402-408, 2001.

19. Hou Z, Guo K, Sun X, Hu F, Chen Q, Luo X, Wang G, Hu J and Sun L: TRIB2 functions as novel oncogene in colorectal cancer by blocking cellular senescence through AP4/p21 signaling. Mol Cancer 17: 172, 2018.

20. Feng ZY, Xu XH, Cen DZ, Luo CY and Wu SB: miR-590-3p promotes colon cancer cell proliferation via Wnt/ $\beta$-catenin signaling pathway by inhibiting WIF1 and DKK1. Eur Rev Med Pharmacol Sci 21: 4844-4852, 2017.

21. Zhang Y, Zhang L, Zhang G, Li S, Duan J, Cheng J, Ding G, Zhou $\mathrm{C}$, Zhang J, Luo P, et al: Osteosarcoma metastasis: Prospective role of ezrin. Tumour Biol 35: 5055-5059, 2014.

22. Calin GA, Sevignani C, Dumitru CD, Hyslop T, Noch E, Yendamuri S, Shimizu M, Rattan S, Bullrich F, Negrini M and Croce CM: Human microRNA genes are frequently located at fragile sites and genomic regions involved in cancers. Proc Natl Acad Sci USA 101: 2999-3004, 2004.

23. Li SL, Gao HL, Lv XK, Hei YR, Li PZ, Zhang JX and Lu N: MicroRNA-124 inhibits cell invasion and epithelial-mesenchymal transition by directly repressing Snail2 in gastric cancer. Eur Rev Med Pharmacol Sci 21: 3389-3396, 2017.

24. Song YX, Sun JX, Zhao JH, Yang YC, Shi JX, Wu ZH, Chen XW, Gao P, Miao ZF and Wang ZN: Non-coding RNAs participate in the regulatory network of CLDN4 via ceRNA mediated miRNA evasion. Nat Commun 8: 289, 2017.
25. Tang L, Hao ZP, Deng Y, Zhang P, Fu XN and Zhang N: MiR-29a-3p suppresses the proliferation, migration and invasion of esophageal squamous cell carcinoma by targeting IGF-1. Int J Clin Exp Pathol 10: 1293-1302, 2017.

26. Liu C, Liu Z, Li X, Tang X, He J and Lu S: MicroRNA-1297 contributes to tumor growth of human breast cancer by targeting PTEN/PI3K/AKT signaling. Oncol Rep 38: 2435-2443, 2017.

27. Ring A, Kim YM and Kahn M: Wnt/catenin signaling in adult stem cell physiology and disease. Stem Cell Rev 10: 512-525, 2014.

28. Stewart DJ, Chang DW, Ye Y, Spitz M, Lu C, Shu X, Wampfler JA, Marks RS, Garces YI, Yang P and Wu X: Wnt signaling pathway pharmacogenetics in non-small cell lung cancer. Pharmacogenom J 14: 509-522, 2014.

29. Dai G, Zheng D, Wang Q, Yang J, Liu G, Song Q, Sun X, Tao C, $\mathrm{Hu} \mathrm{Q}$, Gao T, et al: Baicalein inhibits progression of osteosarcoma cells through inactivation of the Wnt/ $\beta$-catenin signaling pathway. Oncotarget 8: 86098-86116, 2017.

30. MacDonald BT, Tamai K and He X: Wnt/beta-catenin signaling: Components, mechanisms, and diseases. Dev Cell 17: 9-26, 2009

31. Zhang H, Hu B, Wang Z, Zhang F, Wei H and Li L: miR-181c contributes to cisplatin resistance in non-small cell lung cancer cells by targeting Wnt inhibition factor 1 . Cancer Chemother Pharmacol 80: 973-984, 2017.

32. Deng X, Hou C, Wang H, Liang T and Zhu L: Hypermethylation of WIF1 and its inhibitory role in the tumor growth of endometrial adenocarcinoma. Mol Med Rep 16: 7497-7503, 2017.

33. Rubin EM, Guo Y, Tu K, Xie J, Zi X and Hoang BH: Wnt inhibitory factor 1 decreases tumorigenesis and metastasis in osteosarcoma. Mol Cancer Ther 9: 731-741, 2010. 